\title{
EXPLOSION PROBLEMS FOR SYMMETRIC DIFFUSION PROCESSES
}

\author{
KANJI ICHIHARA
}

\begin{abstract}
We discuss the explosion problem for a symmetric diffusion process. Hasminskii's idea cannot be applied to this case. Instead, the theory of Dirichlet forms is employed to obtain criteria for conservativeness and explosion of the process. The fundamental criteria are given in terms of the $\alpha$-equilibrium potentials and $\alpha$-capacities of the unit ball centered at the origin. They are applied to obtain sufficient conditions on the coefficients of the infinitesimal generator for conservativeness and explosion.
\end{abstract}

1. Introduction. Let $\left(X_{t}, \zeta, P_{x}\right), x \in \mathbf{R}^{n}$, be a diffusion process on the Euclidean space $\mathbf{R}^{n}$, where $\zeta$ is the lifetime of $X_{t}$, i.e. $\lim _{t \rightarrow \zeta(w)} X_{t}(w)=\infty$ if $\varsigma(w)<+\infty$.

It is very important from the viewpoint of applications to see whether the process $X_{t}$ is conservative or explosive. Such conditions for one-dimensional diffusion processes have been established by Feller $[\mathbf{1}]$ in connection with the classification of boundary points. His conditions are given in terms of the scale and speed measures. In multidimensional cases, Hasminskii [4] has obtained sufficient conditions for conservativeness and explosion of diffusion processes which are constructed by means of Itô's stochastic differential equations (see also McKean [11]).

In this paper we shall investigate these properties for symmetric diffusion processes. Hasminskii's idea is not applicable to our cases because the infinitesimal generator of our process cannot necessarily be expressed in the following form:

$$
\sum_{i, j=1}^{n} a_{i j} \frac{\partial^{2}}{\partial x_{i} \partial x_{j}}+\sum_{i=1}^{n} b_{i} \frac{\partial}{\partial x_{i}} .
$$

However, we can make use of the theory of Dirichlet forms in order to get conditions for conservativeness and explosion. In $\S 2$, we shall prove a few general criteria for these properties. The criteria are stated in terms of the $\alpha(\geq 0)$-equilibrium potentials and $\alpha$-capacities of the unit ball centered at the origin. In $\S \S 3$ and 4, these criteria are applied to obtain conditions on coefficients for conservativeness and explosion. $\S 3$ is devoted to the study of diffusions associated with strictly elliptic differential operators of selfadjoint form with measurable coefficients. Timechanged processes and processes with singular energy measures will be treated in $\S 4$.

The main results for diffusions associated with differential operators are summarized in the previous note $[\mathbf{7}]$.

Received by the editors August 20, 1985 .

1980 Mathematics Subject Classification (1985 Revision). Primary 60J60. 
2. Fundamental criteria. Let $m$ be a positive Radon measure on $\mathbf{R}^{n}$ strictly positive for each nonempty open set. ( , ) denotes the inner product in the real $L^{2}$-space $L^{2}\left(\mathbf{R}^{n}, m\right)$. Let $\nu_{i j}, i, j=1, \ldots, n$, be Radon measures on $\mathbf{R}^{n}$ such that for any $\xi \in \mathbf{R}^{n}$ and any compact set $K \subset R^{n}$,

$$
\sum_{i, j=1}^{n} \xi_{i} \xi_{j} \nu_{i j}(K) \geq 0, \quad \nu_{i j}(K)=\nu_{j i}(K), i, j=1, \ldots, n .
$$

Define a nonnegative definite symmetric bilinear form $\mathcal{E}$ by

$$
\mathcal{E}(u, v)=\int_{\mathbf{R}^{n}} \sum_{i, j=1}^{n} \frac{\partial u}{\partial x_{i}} \frac{\partial v}{\partial x_{j}} \nu_{i j}(d x), \quad u, v \in C_{0}^{\infty}\left(\mathbf{R}^{n}\right),
$$

where $C_{0}^{\infty}\left(\mathbf{R}^{n}\right)$ is the set of infinitely differentiable functions with compact support. The bilinear form defined above is called a symmetric form on $L^{2}\left(\mathbf{R}^{n}, m\right)$. Throughout this section, we assume that the symmetric form $\mathcal{E}$ on $L^{2}\left(\mathbf{R}^{n}, m\right)$ is closable. Thus the smallest closed extension $\overline{\mathcal{E}}$ of $\mathcal{E}$ is a regular Dirichlet form. Consequently, there exists an $m$-symmetric diffusion process $\left(X_{t}, \zeta, P_{x}\right), x \in \mathbf{R}^{n}$, whose Dirichlet form is the given one $\overline{\mathcal{E}}$, where $\varsigma$ denotes the lifetime of the process $X_{t}$.

Note that the diffusion is unique up to the equivalence. See Fukushima [2] for the details on existence and uniqueness of symmetric diffusions.

Let $G_{\alpha}(x, d y)$ be the $\alpha$-order Green measure defined by

$$
G_{\alpha}(x, d y)=\int_{0}^{\infty} e^{-\alpha t} P(t, x, d y) d t
$$

where $P(t, x, d y)$ is the transition probability of $X_{t}$. We now introduce the following conditions (H.1) and (H.2).

(H.1) $G_{\alpha}(x, \cdot), x \in \mathbf{R}^{n}$, are equivalent to each other.

(H.2) The functions $E_{x}\left[e^{-\alpha \varsigma}\right], \alpha>0$, and $P_{x}[\varsigma<+\infty]$ are continuous in $\mathbf{R}^{n}$.

Let $B_{n}$ denote the closed unit ball centered at the origin, i.e., $B_{n}=\{x \in$ $\left.\mathbf{R}^{n}|| x \mid \leq 1\right\}$. Let us introduce the first hitting time $\tau_{0}$ of $B_{n}$ by $X_{t}$, i.e.

$$
\tau_{0}= \begin{cases}\inf \left\{t \geq 0|| X_{t} \mid \leq 1\right\} & \text { if such } t \text { exists, } \\ +\infty & \text { otherwise. }\end{cases}
$$

The $\alpha$-equilibrium potential $e_{\alpha}(x)$ is defined by

$$
e_{\alpha}(x)= \begin{cases}E_{x}\left[e^{-\alpha \tau_{0}}\right], & \alpha>0 \\ P_{x}\left[\tau_{0}<\zeta\right], & \alpha=0\end{cases}
$$

for all $x \in \mathbf{R}^{n}$. Set

$$
\mathcal{E}_{\alpha}(u, v)=\mathcal{E}(u, v)+\alpha(u, v), \quad \alpha \geq 0,
$$

and denote by $\mathcal{F}_{\alpha}=\mathcal{F}_{\alpha}\left(\mathbf{R}^{n}\right)$ the closure of $C_{0}^{\infty}\left(\mathbf{R}^{n}\right)$ with respect to the norm $\mathcal{E}_{\alpha}$. It is well known that $\left(\mathcal{F}_{\alpha}, \mathcal{E}_{\alpha}\right)$ for $\alpha>0$ is a Hilbert space and that $\left(\mathcal{F}_{0}, \mathcal{E}_{0}\right)$ is a Hilbert space if and only if $X_{t}$ is transient. See Fukushima [2, Chapter 1]. Let us now define the $\alpha$-capacity $C_{\alpha}\left(B_{n}\right)$ of the unit ball $B_{n}$ as follows. For $\alpha>0$,

$$
C_{\alpha}\left(B_{n}\right)=\inf _{\substack{u \in \mathcal{F}_{\alpha} \\ u \geq 1, \text { a.e. on } B_{n}}} \mathcal{E}_{0}(u, u)
$$


and for $\alpha=0$ and $X_{t}$ transient,

$$
C_{0}\left(B_{n}\right)=\inf _{\substack{u \in \mathcal{F}_{0} \\ u \geq 1, \text { a.e. on } B_{n}}} \mathcal{E}_{0}(u, u) .
$$

We have the following lemma.

LEMMA 2.1 (FUKUShima [2]). For each $\alpha>0, e_{\alpha} \in \mathcal{F}_{\alpha}$ and

$$
C_{\alpha}\left(B_{n}\right)=\mathcal{E}_{\alpha}\left(e_{\alpha}, e_{\alpha}\right) \text {. }
$$

Moreover, if $X_{t}$ is transient, $e_{0} \in \mathcal{F}_{0}$ and

$$
C_{0}\left(B_{n}\right)=\varepsilon_{0}\left(e_{0}, e_{0}\right)
$$

REMARK 2.1. A simple variational technique shows that, under the same conditions as in Lemma $2.1, \mathcal{E}_{\alpha}\left(e_{\alpha}, u\right) \geq 0$ for any $u \in \mathcal{F}_{\alpha}$ with $u \geq 0, m$-a.e. on $B_{n}$. In particular, $\mathcal{E}_{\alpha}\left(e_{\alpha}, u\right)=0$ for any $u \in \mathcal{F}_{\alpha}$ with $u=0, m$-a.e. on $B_{n}$.

We are now in a position to state fundamental criteria for conservativeness and explosion of the diffusion process $X_{t}$.

THEOREM A. Suppose the hypothesis (H.1) is satisfied. Then the following statements are equivalent.

(1) $X_{t}$ is conservative, i.e. $P .[\varsigma=+\infty]=1$ on $\mathbf{R}^{n}$.

(2) For some $\alpha>0$,

$$
\int_{\mathbf{R}^{n}} \alpha e_{\alpha}(x) m(d x)=C_{\alpha}\left(B_{n}\right)
$$

(3) For all $\alpha>0$,

$$
\int_{\mathbf{R}^{n}} \alpha e_{\alpha}(x) m(d x)=C_{\alpha}\left(B_{n}\right)
$$

THEOREM B. Suppose the hypothesis (H.1) is satisfied. If $X_{t}$ is transient and if

$$
\int_{\mathbf{R}^{n}} e_{0}(x) m(d x)<+\infty
$$

then the explosion of the process $X_{t}$ is sure, i.e.

$$
P .[\varsigma<+\infty]=1 \text { on } \mathbf{R}^{n} \text {. }
$$

If, in addition, (H.2) is satisfied, we obtain a more precise result.

THEOREM C. Suppose the hypotheses (H.1) and (H.2) are satisfied. If $X_{t}$ is transient, then the limit of $\int_{\mathbf{R}^{n}} \alpha e_{\alpha}(x) m(d x)$ as $\alpha \downarrow 0$ exists and

$$
\lim _{\alpha \downarrow 0} \int_{\mathbf{R}^{n}} \alpha e_{\alpha}(x) m(d x) \begin{cases}=C_{0}\left(B_{n}\right), & \text { when } P .[\zeta=+\infty]-=1 \text { on } \mathbf{R}^{n}, \\ \in\left(0, C_{0}\left(B_{n}\right)\right), & \text { when } 0<P .[\zeta=+\infty]<1 \text { on } \mathbf{R}^{n} \\ =0, & \text { when } P .[\zeta=+\infty]=0 \text { on } \mathbf{R}^{n} .\end{cases}
$$

The following proposition is a simple consequence of Theorem $\mathrm{B}$.

PROPOSITION 2.1. Suppose the hypothesis (H.1) is satisfied. If $X_{t}$ is transient and if the total mass of the speed measure is finite, i.e. $m\left(\mathbf{R}^{n}\right)<+\infty$, then the explosion of the process $X_{t}$ is sure.

In the proofs of the fundamental criteria for conservativeness and explosion, the following lemma plays an important role. 
Lemma 2.2 (Fukushima [2]). (1) For each $\alpha>0$, there exists a unique finite measure $\mu_{\alpha}$ with its support in $B_{n}$ such that

$$
\mathcal{E}_{\alpha}\left(e_{\alpha}, u\right)=\int_{B_{n}} \tilde{u}(x) \mu_{\alpha}(d x), \quad u \in \mathcal{F}_{\alpha} .
$$

(2) For $\alpha=0$ and $X_{t}$ transient, there exists a unique finite measure $\mu_{0}$ with its support in $\partial B_{n}$, the boundary of $B_{n}$, such that

$$
\mathcal{E}_{0}\left(e_{0}, u\right)=\int_{\partial B_{n}} \tilde{u}(x) \mu_{0}(d x), \quad u \in \mathcal{F}_{0} .
$$

Here $\tilde{u}$ denotes any quasicontinuous modification of $u \in \mathcal{F}_{\alpha}$.

REMARK 2.2. The finite measure $\mu_{\alpha}$ in the above lemma is called the $\alpha$ equilibrium measure of $B_{n}$. The total mass of the $\alpha$-equilibrium measure is equal to the $\alpha$-capacity of $B_{n}$, i.e.

$$
\mu_{\alpha}\left(B_{n}\right)=C_{\alpha}\left(B_{n}\right), \quad \alpha>0, \quad \text { and } \quad \mu_{0}\left(\partial B_{n}\right)=C_{0}\left(B_{n}\right) .
$$

PROOF OF THEOREM A. Let $f$ be an infinitely differentiable function with compact support. Define the $\alpha$-order Green operator $G_{\alpha}$ by

$$
G_{\alpha} f(x)=\int_{\mathbf{R}^{n}} G_{\alpha}(x, d y) f(y) .
$$

Note that $G_{\alpha} f$ is quasicontinuous. See Theorem 4.3.3, Fukushima [2]. Then

$$
\int_{\mathbf{R}^{n}} \alpha e_{\alpha}(x) f(x) m(d x)=\alpha\left(e_{\alpha}, f\right)=\alpha \mathcal{E}_{\alpha}\left(e_{\alpha}, G_{\alpha} f\right) .
$$

Applying Lemma 2.2 to $u=G_{\alpha} f$, we obtain

$$
\int_{\mathbf{R}^{n}} \alpha e_{\alpha}(x) f(x) m(d x)=\alpha \int_{B_{n}} G_{\alpha} f(x) \mu_{\alpha}(d x) .
$$

Taking the limits of both sides of $(2.1)$ as $f \uparrow 1$ gives

$$
\int_{\mathbf{R}^{n}} \alpha e_{\alpha}(x) m(d x)=\int_{B_{n}} \alpha G_{\alpha} 1(x) \mu_{\alpha}(d x) .
$$

Since

$$
G_{\alpha} 1(x)=\int_{0}^{+\infty} e^{-\alpha t} E_{x}[1 ; t<\varsigma] d t=E_{x}\left[\int_{0}^{\varsigma} e^{-\alpha t} d t\right]=\frac{1}{\alpha}\left\{1-E_{x}\left[e^{-\alpha \varsigma}\right]\right\},
$$

where $E_{x}$ denotes the expectation with respect to the probability measure $P_{x}$, we obtain

$$
\int_{\mathbf{R}^{n}} \alpha e_{\alpha}(x) m(d x)=\int_{B_{n}} \mu_{\alpha}(d x)\left\{1-E_{x}\left[e^{-\alpha \varsigma}\right]\right\}=C_{\alpha}\left(B_{n}\right)-\int_{B_{n}} E_{x}\left[e^{-\alpha \varsigma}\right] \mu_{\alpha}(d x) .
$$

Now the implication (1) $\Rightarrow(2)$ and (3) is obvious. Suppose that the condition (2) holds for some $\alpha>0$. Then we have

$$
\int_{B_{n}} E_{x}\left[e^{-\alpha \varsigma}\right] \mu_{\alpha}(d x)=0
$$


Consequently, $E_{x}\left[e^{-\alpha \varsigma}\right]=0 \mu_{\alpha}$-a.e. on $B_{n}$. Thus $P_{x}[\zeta=+\infty]=1 \mu_{\alpha}$-a.e. on $B_{n}$. Fix a point $x_{0} \in B_{n}$ such that

$$
P_{x_{0}}[\varsigma=+\infty]=1
$$

Set $q_{0}(x)=P_{x}[\varsigma=+\infty], x \in \mathbf{R}^{n}$. The Markov property shows that

$$
\begin{aligned}
q_{0}(x) & =P_{x}[\zeta=+\infty]=P_{x}[t<\zeta, \zeta=+\infty] \\
& =E_{x}\left[P_{X_{t}}[\zeta=+\infty] ; t<\zeta\right]=E_{x}\left[q_{0}\left(X_{t}\right) ; t<\zeta\right] .
\end{aligned}
$$

Perform the Laplace transform of both sides of (2.3) to obtain

$$
(1 / \alpha) q_{0}(x)=G_{\alpha} q_{0}(x)
$$

From (2.2), we have $q_{0}(x)=1, G_{\alpha}\left(x_{0}, \cdot\right)$-a.e. on $\mathbf{R}^{n}$. Taking the hypothesis (H.1) into consideration, we obtain

$$
q_{0}(x)=\alpha G_{\alpha} q_{0}(x)=\alpha G_{\alpha} 1(x)
$$

for all $x \in \mathbf{R}^{n}$. Therefore

$$
P_{x}[\zeta=+\infty]=1-E_{x}\left[e^{-\alpha \varsigma}\right], \quad x \in \mathbf{R}^{n} .
$$

This implies $P$. $[\zeta=+\infty]=1$ on $\mathbf{R}^{n}$. Q.E.D.

PROOF OF THEOREM B. By Theorem 1.5.3, Fukushima [2], we have for any infinitely differentiable function $f(x)$ with compact support,

$$
\int_{\mathbf{R}^{n}} e_{0}(x) f(x) m(d x)=\left(e_{0}, f\right)=\mathcal{E}_{0}\left(G_{0} f, e_{0}\right) .
$$

Applying Lemma 2.2 to $G_{0} f$, we obtain

$$
\int_{\mathbf{R}^{n}} e_{0}(x) f(x) m(d x)=\int_{\partial B_{n}} G_{0} f(x) \mu_{0}(d x) .
$$

Here $G_{0}$ is the 0 -order Green operator. Letting $f \uparrow 1$, it follows that

$$
\int_{\mathbf{R}^{n}} e_{0}(x) m(d x)=\int_{\partial B_{n}} G_{0} 1(x) \mu_{0}(d x) .
$$

Since $G_{0} 1(x)=E_{x}\left[\int_{0}^{\varsigma} d t\right]=E_{x}[\zeta]$, we obtain

$$
\infty>\int_{\mathbf{R}^{n}} e_{0}(x) m(d x)=\int_{\partial B_{n}} E_{x}[\zeta] \mu_{0}(d x),
$$

which implies $E_{x}[\zeta]<\infty \mu_{0}$-a.e. on $\partial B_{n}$. Therefore,

$$
P_{x}[\zeta<+\infty]=1, \quad \mu_{0} \text {-a.e. on } \partial B_{n} .
$$

As was shown in the proof of Theorem A, we have $q_{0}(x)=\alpha G_{\alpha} q_{0}(x), \alpha>0$, with $q_{0}(x)=P_{x}[\varsigma=+\infty]$. From (2.4), we have at least one point $x_{0}$ on $\partial B_{n}$ such that $q_{0}\left(x_{0}\right)=P_{x_{0}}[\zeta=+\infty]=0$. Hence

$$
0=q_{0}\left(x_{0}\right)=\alpha G_{\alpha} q_{0}\left(x_{0}\right) .
$$

Thus $q_{0}(x)=0, G_{\alpha}\left(x_{0}, \cdot\right)$-a.e. on $\mathbf{R}^{n}$. The hypothesis (H.1) gives

$$
q_{0}(x)=\alpha G_{\alpha} q_{0}(x)=\alpha G_{\alpha} 0(x)=0
$$

for all $x \in \mathbf{R}^{n}$, which completes the proof of Theorem B. Q.E.D.

In order to prove Theorem $\mathrm{C}$, we need the following lemmas. 
Lemma 2.3. Suppose $X_{t}$ is transient. Then

$$
\lim _{\alpha \downarrow 0} \mathcal{E}_{0}\left(e_{\alpha}-e_{0}, e_{\alpha}-e_{0}\right)=0 .
$$

In particular, we have $\lim _{\alpha \downarrow 0} C_{\alpha}\left(B_{n}\right)=C_{0}\left(B_{n}\right)$.

ProOF. First note that $\mathcal{E}_{\alpha}\left(e_{\alpha}, e_{\alpha}\right)=C_{\alpha}\left(B_{n}\right)$ is monotone nonincreasing as $\alpha \downarrow 0$ by virtue of the minimizing property of $e_{\alpha}$. Thus $\mathcal{E}_{\alpha}\left(e_{\alpha}, e_{\alpha}\right)$ is convergent as $\alpha \downarrow 0$. We have, for $\alpha>\alpha^{\prime}$,

$$
\begin{aligned}
\mathcal{E}_{0}\left(e_{\alpha}-e_{\alpha^{\prime}}, e_{\alpha}-e_{\alpha^{\prime}}\right) & \leq \mathcal{E}_{\alpha^{\prime}}\left(e_{\alpha}-e_{\alpha^{\prime}}, e_{\alpha}-e_{\alpha^{\prime}}\right) \\
& =\mathcal{E}_{\alpha^{\prime}}\left(e_{\alpha^{\prime}}, e_{\alpha^{\prime}}\right)+\mathcal{E}_{\alpha^{\prime}}\left(e_{\alpha}, e_{\alpha}\right)-2 \mathcal{E}_{\alpha^{\prime}}\left(e_{\alpha}, e_{\alpha^{\prime}}\right) .
\end{aligned}
$$

From Remark 2.1, it follows that $\mathcal{E}_{\alpha^{\prime}}\left(e_{\alpha^{\prime}}, e_{\alpha^{\prime}}-e_{\alpha}\right)=0$. Thus $\mathcal{E}_{\alpha^{\prime}}\left(e_{\alpha^{\prime}}, e_{\alpha^{\prime}}\right)=$ $\mathcal{E}_{\alpha^{\prime}}\left(e_{\alpha^{\prime}}, e_{\alpha}\right)$. Consequently, we obtain

$$
\mathcal{E}_{0}\left(e_{\alpha}-e_{\alpha^{\prime}}, e_{\alpha}-e_{\alpha^{\prime}}\right) \leq \mathcal{E}_{\alpha}\left(e_{\alpha}, e_{\alpha}\right)-\mathcal{E}_{\alpha^{\prime}}\left(e_{\alpha^{\prime}}, e_{\alpha^{\prime}}\right),
$$

which tends to zero as $\alpha, \alpha^{\prime} \downarrow 0$. This implies that $\left\{e_{\alpha}, \alpha>0\right\}$ is a Cauchy sequence in $\left(\mathcal{F}_{0}, \mathcal{E}_{0}\right)$ as $\alpha \downarrow 0$. Since $\left(\mathcal{F}_{0}, \mathcal{E}_{0}\right)$ is a Hilbert space, there exists a unique element $\tilde{e}_{0}$ in $\mathcal{F}_{0}$ to which $e_{\alpha}$ converges as $\alpha \downarrow 0$. On the other hand, $e_{\alpha}(x)$ approaches $e_{0}(x)$ pointwise on $\mathbf{R}^{n}$ as $\alpha \downarrow 0$. Hence $\tilde{e}_{0}$ must coincide with $e_{0}$. Thus we obtain

$$
\lim _{\alpha \downarrow 0} \mathcal{E}_{0}\left(e_{\alpha}-e_{0}, e_{\alpha}-e_{0}\right)=0 .
$$

It is obvious from Remark 2.1 that, for a function $f(x)$ in $C_{0}^{\infty}\left(\mathbf{R}^{n}\right)$ with $f=1$ on $B_{n}$,

$$
C_{\alpha}\left(B_{n}\right)=\mathcal{E}_{\alpha}\left(e_{\alpha}, f\right), \quad \alpha \geq 0 .
$$

Since $f$ has compact support, (2.5) together with (2.6) gives that

$$
\lim _{\alpha \downarrow 0} C_{\alpha}\left(B_{n}\right)=\lim _{\alpha \downarrow 0} \mathcal{E}_{\alpha}\left(e_{\alpha}, f\right)=\mathcal{E}_{0}\left(e_{0}, f\right)=C_{0}\left(B_{n}\right) . \quad \text { Q.E.D. }
$$

Lemma 2.4. Suppose $X_{t}$ is transient. Then for any continuous function $u(x)$ on $\mathbf{R}^{n}$,

$$
\lim _{\alpha \downarrow 0} \int_{B_{n}} u(x) \mu_{\alpha}(d x)=\int_{\partial B_{n}} u(x) \mu_{0}(d x) .
$$

ProOF. By virtue of Lemmas 2.2 and 2.3, it follows that

$$
\lim _{\alpha \downarrow 0} \int_{B_{n}} u(x) \mu_{\alpha}(d x)=\int_{\partial B_{n}} u(x) \mu_{0}(d x)
$$

for any $u \in C_{0}^{\infty}\left(\mathbf{R}^{n}\right)$. Since any continuous function can be uniformly approximated on $B_{n}$ by infinitely differentiable functions, the assertion in Lemma 2.4 is valid. Q.E.D.

ProOF OF THEOREM C. As was shown in the proof of Theorem A, it holds that

$$
\int_{\mathbf{R}^{n}} \alpha e_{\alpha}(x) m(d x)=\int_{B_{n}} \alpha G_{\alpha} 1(x) \mu_{\alpha}(d x) .
$$

Note that $\alpha G_{\alpha} 1(x)=1-E_{x}\left[e^{-\alpha \varsigma}\right]$ is monotone nonincreasing and is convergent as $\alpha \downarrow 0$ to $1-P_{x}[\zeta<+\infty]$. Consequently, from the hypothesis (H.2), the convergence 
$\alpha G_{\alpha} 1(x) \downarrow 1-P_{x}[\varsigma<+\infty]$ is uniform on each compact subset of $\mathbf{R}^{n}$. This together with Lemma 2.4 gives

$$
\lim _{\alpha \downarrow 0} \int_{\mathbf{R}^{n}} \alpha e_{\alpha}(x) m(d x)=\lim _{\alpha \downarrow 0} \int_{B_{n}} \alpha G_{\alpha} 1(x) \mu_{\alpha}(d x)=\int_{\partial B_{n}} q_{0}(x) \mu_{0}(d x) .
$$

Suppose that $\lim _{\alpha \downarrow 0} \int_{\mathbf{R}^{n}} \alpha e_{\alpha}(x) m(d x)=C_{0}\left(B_{n}\right)$. Then we have $\int_{\partial B_{n}} q_{0}(x) \mu_{0}(d x)$ $=C_{0}\left(B_{n}\right)$. Since the total measure of $\mu_{0}$ is the capacity $C_{0}\left(B_{n}\right)$, the above equality gives $q_{0}(x)=1, \mu_{0}$-a.e. on $\partial B_{n}$. By the same argument as in the proof of Theorem A, we can show that $q_{0}(x)=P_{x}[\zeta=+\infty]$ is identically equal to 1 on $\mathbf{R}^{n}$. We next consider the case

$$
\lim _{\alpha \downarrow 0} \int_{\mathbf{R}^{n}} \alpha e_{\alpha}(x) m(d x)=0 .
$$

Then $\int_{\partial B_{n}} q_{0}(x) \mu_{0}(d x)=0$, which gives $q_{0}(x)=0, \mu_{0}$-a.e. on $\partial B_{n}$. Then, by the same argument as in the first case, it follows that $q_{0}(x)=0$ for all $x \in \mathbf{R}^{n}$. The rest is obvious from the conclusions for the first two cases. Q.E.D.

\section{Strictly elliptic differential operator with measurable coefficients.}

In this section we will discuss the explosion problems for symmetric diffusion processes corresponding to Dirichlet forms endowed with measures $\nu_{i j}, i, j=1, \ldots, n$, and $m$ absolutely continuous with respect to Lebesgue measure $d x$, i.e.,

$$
\begin{aligned}
\nu_{i j}(d x) & =a_{i j}(x) d x, \quad i, j=1, \ldots, n, \\
m(d x) & =b(x) d x .
\end{aligned}
$$

We assume that for each nonempty compact subset $K$ of $\mathbf{R}^{n}$ there exists a positive constant $\lambda=\lambda(K)>1$ such that

$$
\begin{aligned}
& \lambda^{-1} \sum_{i=1}^{n} \xi_{i}^{2} \leq \sum_{i, j=1}^{n} a_{i j}(x) \xi_{i} \xi_{j} \leq \lambda \sum_{i=1}^{n} \xi_{i}^{2} \\
& \lambda^{-1} \leq b(x) \leq \lambda
\end{aligned}
$$

for all $x \in K$ and $\xi=\left(\xi_{i}\right)_{i=1, \ldots, n} \in \mathbf{R}^{n}$. It is well known that for the above $\nu_{i j}$ and $m$, the symmetric form $\mathcal{E}$ on $L^{2}\left(\mathbf{R}^{n}, m\right)$ is closable. Consequently, there exists an $m$-symmetric diffusion process except for a set of zero capacity whose Dirichlet form coincides with the smallest closed extension $\overline{\mathcal{E}}$. However, in the present case, we have a stronger result. In fact, there exists a unique minimal diffusion process $\left(X_{t}, \zeta, P_{x}\right), x \in \mathbf{R}^{n}$, whose resolvent $R_{\lambda}^{G}$ restricted to a bounded open set $G$ is continuous in the following sense: for any $f \in L^{p}(G, m)$ with $p>n, R_{\lambda}^{G} f(x)$ is continuous on $\bar{G}$. See, for example, Tomisaki $[\mathbf{1 6}]$. Note that the infinitesimal generator of this diffusion coincides with the following strictly elliptic differential operator of selfadjoint form with measurable coefficients:

$$
L=\frac{1}{b} \sum_{i, j=1}^{n} \frac{\partial}{\partial x_{i}}\left(a_{i j} \frac{\partial}{\partial x_{j}}\right)
$$

REMARK 3.1. For the diffusion, the hypotheses (H.1) and (H.2) are satisfied. Indeed, (H.1) is obvious from Theorem 9.2, Tomisaki [16]. In order to show 
(H.2), we employ some results in Gilbarg and Trudinger [3]. Consider the Dirichlet problem

$$
\begin{cases}\frac{1}{b} \sum_{i, j=1}^{n} \frac{\partial}{\partial x_{i}}\left(a_{i j} \frac{\partial}{\partial x_{j}} u\right)-\alpha u=-\alpha & \text { in }|x|<\rho, \\ u(x)=0 & \text { on }|x|=\rho,\end{cases}
$$

where $\alpha$ and $\rho$ are positive numbers. Then Theorems 8.30 and 8.22 in Gilbarg and Trudinger $[\mathbf{3}]$ ensure that there exists a unique solution $u_{\alpha}^{\rho}$ which is locally Hölder continuous in $|x|<\rho$. Let $G_{\alpha}^{\rho}$ denote the $\alpha$-order Green operator of the minimal diffusion process in $|x|<\rho$ associated with the operator

$$
\frac{1}{b} \sum_{i, j=1}^{n} \frac{\partial}{\partial x_{i}}\left(a_{i j} \frac{\partial}{\partial x_{j}}\right) .
$$

It is easily seen that $u_{\alpha}^{\rho}(x)=\alpha G_{\alpha}^{\rho} 1(x)$. Theorem 8.22 asserts that $u_{\alpha}^{\rho}, \alpha \leq 1$, is uniformly Hölder continuous on each compact subset in $|x|<\rho$, i.e., for each compact subset $K \subset\{|x|<\rho\}$, there exist positive numbers $C=C(K, L, n+1)$ and $\beta=\beta(K, L, n+1)<1$ independent of $\rho$ such that $\left|u_{\alpha}^{\rho}(x)-u_{\alpha}^{\rho}(y)\right| \leq C|x-y|^{\beta}$ for any $x$ and $y$ in $K$. Taking the limit of $u_{\alpha}^{\rho}$ as $\rho \uparrow \infty$, we obtain

$$
\left|\alpha G_{\alpha} 1(x)-\alpha G_{\alpha} 1(y)\right| \leq C|x-y|^{\beta}, \quad x, y \in K,
$$

which implies $E_{x}\left[e^{-\alpha \varsigma}\right]=1-\alpha G_{\alpha} 1(x)$ is continuous. Furthermore, letting $\alpha \downarrow 0$ in (3.1), we obtain

$$
\left|P_{x}[\varsigma=+\infty]-P_{y}[\varsigma=+\infty]\right| \leq C|x-y|^{\beta}, \quad x, y \in K .
$$

Therefore $P_{x}[\varsigma<+\infty]=1-P_{x}[\varsigma=+\infty]$ is also continuous. Thus the hypothesis (H.2) is satisfied.

Remark 3.1 shows that Theorems A, B, and C are valid for the above diffusion process. The main purpose of this section is to give conditions on the coefficients $a_{i j}$ and $b$ for conservativeness and explosion. Set

$$
A(x)=\left(a_{i j}(x)\right)_{i, j=1, \ldots, n} \quad \text { for } x \in \mathbf{R}^{n}
$$

and define

$$
\begin{aligned}
& A_{+}(r)=\int_{S^{n-1}}\langle A(r \sigma) \sigma, \sigma\rangle d \sigma \quad \text { for } r>0, \\
& B_{+}(r)=\sup _{\sigma \in S^{n-1}}\left\{\frac{\langle A(r \sigma) \sigma, \sigma\rangle}{b(r \sigma)}\right\} \quad \text { for } r>0,
\end{aligned}
$$

where $d \sigma$ is the uniform measure on the unit sphere $S^{n-1}$ and $\langle$,$\rangle is the inner$ product on $\mathbf{R}^{n}$. We have the following theorem for conservativeness.

THEOREM 3.1. If for some $\alpha>0$,

$$
\lim _{r \uparrow+\infty} \frac{\exp \left\{-\int_{1}^{r} 2 \sqrt{\alpha} / \sqrt{B_{+}(s)} d s\right\}}{\int_{r}^{+\infty} s^{1-n} A_{+}(s)^{-1} d s}=0,
$$

then the process $X_{t}$ is conservative.

It should be remarked that Ichihara $[6]$ has shown that $X_{t}$ is recurrent if the denominator in the assumption of Theorem 3.1 is infinite. Thus, in this case, the process is conservative.

A function $u$ is said to belong to $H_{\text {loc }}^{1,2}\left(\mathbf{R}^{n}\right)$ if $u \in L_{\text {loc }}^{2}\left(\mathbf{R}^{n}\right)$ and if its distribution derivatives $\partial u / \partial x_{i} \in L_{\text {loc }}^{2}\left(\mathbf{R}^{n}\right)$. We need the following lemma. 
LEMMA 3.1. Let $\mathbf{f}=\left(f_{i}\right)_{i=1, \ldots, n}$ where $f_{i} \in H_{\text {loc }}^{1,2}\left(\mathbf{R}^{n}\right)$. Define

$$
\operatorname{div} \mathbf{f}=\sum_{i=1}^{n} \frac{\partial f_{i}}{\partial x_{i}} \quad \text { and } \quad F(r)=\int_{|x|=r}\langle\mathbf{f}, \mathbf{n}\rangle d S .
$$

Then the derivative of $F(r)$ in the distribution sense is given by

$$
\frac{d}{d r} F(r)=\int_{|x|=r} \operatorname{div} \mathbf{f} d S \quad \text { for } \text { a.a. } r>0 .
$$

Here $\mathbf{n}=x /|x|$ and $d S$ is the surface element on $|x|=r$.

PROOF. Let $\phi$ be an infinitely differentiable function with compact support. Furthermore, suppose $\phi$ is rotationally symmetric about the origin. We now compute

$$
\int_{\mathbf{R}^{n}} \operatorname{div} \mathbf{f} \phi d x=-\int_{\mathbf{R}^{n}}\langle\mathbf{f}, \operatorname{grad} \phi\rangle d x .
$$

The left-hand side in the above equality is equal to

$$
\int_{0}^{+\infty} \phi(r)\left[\int_{|x|=r} \operatorname{div} \mathbf{f} d S\right] d r
$$

and the right-hand side is

$$
-\int_{0}^{+\infty} \phi^{\prime}(r)\left[\int_{|x|=r}\langle\mathbf{f}, \mathbf{n}\rangle d S\right] d r .
$$

Thus $\int_{0}^{+\infty} \phi(r)\left[\int_{|x|=r} \operatorname{div} \mathbf{f} d S\right] d r=-\int_{0}^{+\infty} \phi^{\prime}(r)\left[\int_{|x|=r}\langle\mathbf{f}, \mathbf{n}\rangle d S\right] d r$. This implies $d F(r) / d r=\int_{|x|=r} \operatorname{div} \mathbf{f} d S$. Q.E.D.

The function $e_{\alpha}$ is a weak solution of the Dirichlet problem

$$
\begin{cases}\frac{1}{b} \sum_{i, j=1}^{n} \frac{\partial}{\partial x_{i}}\left(a_{i j} \frac{\partial}{\partial x_{j}} u\right)=\alpha u & \text { in }|x|>1, \\ u(x)=1 & \text { on }|x|=1 .\end{cases}
$$

Therefore, by an argument similar to that in Remark 3.1, taking the continuous modification if necessary, we may assume that $e_{\alpha}$ is locally Hölder continuous in $|x|>1$. Furthermore, without loss of generality, we may assume that all the coefficients $a_{i j}$ and $b$ are infinitely differentiable in a neighborhood of $S^{n-1}$, because conservativeness and explosion depend only on the behaviors of the coefficients near infinity. Under this assumption, $e_{\alpha}$ is proved to be twice continuously differentiable on $1 \leq|x| \leq 1+\varepsilon_{0}$ with $\varepsilon_{0}$ a positive number. See, for example, Mizohata [13]. We have

$$
C_{\alpha}\left(B_{n}\right)=\int_{\mathbf{R}^{n}}\left\{\left\langle A \nabla e_{\alpha}, \nabla e_{\alpha}\right\rangle+\alpha e_{\alpha}^{2} b\right\} d x
$$

where

$$
\begin{aligned}
\nabla e_{\alpha} & =\left(\frac{\partial e_{\alpha}}{\partial x_{i}}\right)_{i=1, \ldots, n} \\
& =\int_{B_{n}} \alpha b d x+\int_{|x|>1}\left\{\left\langle A \nabla e_{\alpha}, \nabla e_{\alpha}\right\rangle+\alpha e_{\alpha}^{2} b\right\} d x \\
& =\int_{B_{n}} \alpha b d x+\int_{|x|>1}\left\{\left\langle A \nabla e_{\alpha}, \nabla u\right\rangle+\alpha e_{\alpha} u b\right\} d x
\end{aligned}
$$


for $u \in \mathcal{F}_{\alpha}$ with $u=1$ on $B_{n}$. Setting

$$
u=u_{\varepsilon}= \begin{cases}1 & \text { for }|x| \leq 1 \\ 1-(1 / \varepsilon)(|x|-1) & \text { for } 1<|x|<1+\varepsilon \\ 0 & \text { otherwise }\end{cases}
$$

for $\varepsilon>0$, we have

$$
C_{\alpha}\left(B_{n}\right)=\int_{B_{n}} \alpha b d x-\frac{1}{\varepsilon} \int_{1<|x|<1+\varepsilon}\left\langle A \nabla e_{\alpha}, \mathbf{n}\right\rangle d x+\int_{1<|x|<1+\varepsilon} \alpha e_{\alpha} u_{\varepsilon} b d x .
$$

Letting $\varepsilon \downarrow 0$, we obtain

$$
C_{\alpha}\left(B_{n}\right)=\int_{B_{n}} \alpha b d x-\int_{|x|=1}\left\langle A \nabla e_{\alpha}, \mathbf{n}\right\rangle d S
$$

Applying Lemma 3.1 to $\mathbf{f}=A \nabla e_{\alpha}$, we obtain

$$
\begin{aligned}
\int_{1<|x|<r} \alpha e_{\alpha} b d x & =\int_{1<|x|<r} \operatorname{div}\left(A \nabla e_{\alpha}\right) d x=\int_{1}^{r} d u \int_{|x|=u} \operatorname{div}\left(A \nabla e_{\alpha}\right) d S \\
& =\int_{1}^{r} d u \frac{d}{d u} \int_{|x|=u}\left\langle A \nabla e_{\alpha}, \mathbf{n}\right\rangle d S \\
& =\int_{|x|=r}\left\langle A \nabla e_{\alpha}, \mathbf{n}\right\rangle d S-\int_{|x|=1}\left\langle A \nabla e_{\alpha}, \mathbf{n}\right\rangle d S
\end{aligned}
$$

for a.a. $r>1$. Hence,

$$
\int_{|x|=r}\left\langle A \nabla e_{\alpha}, \mathbf{n}\right\rangle d S, \quad \text { a.a } r>1,
$$

is monotone nondecreasing as $r \uparrow+\infty$. Furthermore it is nonpositive for a.a. $r>1$. Indeed, as was shown in the proof of Theorem A,

$$
C_{\alpha}\left(B_{n}\right) \geq \int_{\mathbf{R}^{n}} \alpha e_{\alpha} b d x \geq \int_{|x|<r} \alpha e_{\alpha} b d x \text { for } r>1 .
$$

Since $C_{\alpha}\left(B_{n}\right)=\int_{B_{n}} \alpha b d x-\int_{|x|=1}\left\langle A \nabla e_{\alpha}, \mathbf{n}\right\rangle d S$ and since

$$
\int_{|x|<r} \alpha e_{\alpha} b d x=\int_{B_{n}} \alpha b d x+\int_{|x|=r}\left\langle A \nabla e_{\alpha}, \mathbf{n}\right\rangle d S-\int_{|x|=1}\left\langle A \nabla e_{\alpha}, \mathbf{n}\right\rangle d S,
$$

we obtain $\int_{|x|=r}\left\langle A \nabla e_{\alpha}, \mathbf{n}\right\rangle d S \leq 0$ for a.a. $r>1$. Define

$$
a_{\alpha}=\lim _{\text {a.a. } r \rightarrow+\infty} \int_{|x|=r}\left\langle A \nabla e_{\alpha}, \mathbf{n}\right\rangle d S .
$$

Taking the limit as $r \uparrow+\infty$, we obtain

$$
\begin{aligned}
& a_{\alpha}-\int_{|x|=1}\left\langle A \nabla e_{\alpha}, \mathbf{n}\right\rangle d S+\int_{B_{n}} \alpha b d x=\int_{\mathbf{R}^{n}} \alpha e_{\alpha} b d x \\
& \leq C_{\alpha}\left(B_{n}\right)=-\int_{|x|=1}\left\langle A \nabla e_{\alpha}, \mathbf{n}\right\rangle d S+\int_{B_{n}} \alpha b d x .
\end{aligned}
$$

Thus we can arrive at the following interesting criterion for conservativeness. 
PROPOSITION 3.1. $X_{t}$ is conservative if and only if $a_{\alpha}=0$.

In order to prove Theorem 3.1, it suffices to verify $a_{\alpha}=0$ under the assumption.

Lemma 3.2. Suppose that $u \in H_{\mathrm{loc}}^{1,2}\left(\mathbf{R}^{n}\right)$ and that $\mathbf{v}=\left(v_{i}\right)_{i=1, \ldots, n}, v_{i} \in$ $H_{\text {loc }}^{1,2}\left(\mathbf{R}^{n}\right)$. Then $\operatorname{div}(u \mathbf{v})=\langle\nabla u, \mathbf{v}\rangle+u \operatorname{div} \mathbf{v}$. Here the derivatives are taken in the distribution sense.

Proof. Let $\psi_{\varepsilon}$ be a mollifier and define $\mathbf{v}_{\varepsilon}=\mathbf{v} * \psi_{\varepsilon}$, where $*$ denotes the convolution. Then $\mathbf{v}_{\varepsilon}$ and $\operatorname{div} \mathbf{v}_{\varepsilon}=(\operatorname{div} \mathbf{v}) * \psi_{\varepsilon}$ converge in $L_{\text {loc }}^{2}\left(\mathbf{R}^{n}\right)$ to $\mathbf{v}$ and $\operatorname{div} \mathbf{v}$ respectively. Thus we have, for any $\phi \in C_{0}^{\infty}\left(\mathbf{R}^{n}\right)$,

$$
\begin{aligned}
\int_{\mathbf{R}^{n}} \operatorname{div}\left(u \mathbf{v}_{\varepsilon}\right) \phi d x & =-\int_{\mathbf{R}^{n}} u\left\langle\mathbf{v}_{\varepsilon}, \nabla \phi\right\rangle d x \\
& \rightarrow-\int_{\mathbf{R}^{n}} u\langle\mathbf{v}, \nabla \phi\rangle d x \quad \text { as } \varepsilon \downarrow 0 \\
& =\int_{\mathbf{R}^{n}} \operatorname{div}(u \mathbf{v}) \phi d x .
\end{aligned}
$$

On the other hand, we have

$$
\begin{aligned}
\int_{\mathbf{R}^{n}} \operatorname{div}\left(u \mathbf{v}_{\varepsilon}\right) \phi d x & =-\int_{\mathbf{R}^{n}} u\left\langle\mathbf{v}_{\varepsilon}, \nabla \phi\right\rangle d x \\
& =-\int_{\mathbf{R}^{n}} u\left\{\operatorname{div}\left(\phi \mathbf{v}_{\varepsilon}\right)-\left(\operatorname{div} \mathbf{v}_{\varepsilon}\right) \phi\right\} d x \\
& =\int_{\mathbf{R}^{n}}\left\langle\nabla u, \mathbf{v}_{\varepsilon}\right\rangle \phi d x+\int_{\mathbf{R}^{n}} u \phi \operatorname{div} \mathbf{v}_{\varepsilon} d x \\
& \rightarrow \int_{\mathbf{R}^{n}}\langle\nabla u, \mathbf{v}\rangle \phi, d x+\int_{\mathbf{R}^{n}} u \phi \operatorname{div} \mathbf{v} d x \quad \text { as } \varepsilon \downarrow 0 .
\end{aligned}
$$

Thus the assertion in Lemma 3.2 follows from (3.3) and (3.4). Q.E.D.

Back to the proof of Theorem 3.1, let $\phi$ be an infinitely differentiable function with compact support such that $\phi$ is rotationally symmetric about the origin.

$$
\int_{\mathbf{R}^{n}} \operatorname{div}\left(e_{\alpha} A \nabla e_{\alpha}\right) \phi d x=-\int_{\mathbf{R}^{n}} e_{\alpha}\left\langle A \nabla e_{\alpha}, \mathbf{n}\right\rangle \phi^{\prime}(|x|) d x .
$$

By Lemma 3.2, the left-hand side is equal to

$$
\int_{\mathbf{R}^{n}}\left\langle\nabla e_{\alpha}, A \nabla e_{\alpha}\right\rangle \phi d x+\int_{\mathbf{R}^{n}} e_{\alpha} \operatorname{div}\left(A \nabla e_{\alpha}\right) \phi d x=\int_{\mathbf{R}^{n}}\left\{\left\langle A \nabla e_{\alpha}, \nabla e_{\alpha}\right\rangle+\alpha e_{\alpha}^{2} b\right\} \phi d x .
$$

Since $\phi$ is a radial function, we have the equality

$$
\begin{aligned}
\int_{0}^{+\infty} \phi(r) d r \int_{|x|=r} & \left\{\left\langle A \nabla e_{\alpha}, \nabla e_{\alpha}\right\rangle+\alpha e_{\alpha}^{2} b\right\} d S \\
= & -\int_{0}^{+\infty} \phi^{\prime}(r) d r \int_{|x|=r} e_{\alpha}\left\langle A \nabla e_{\alpha}, \mathbf{n}\right\rangle d S
\end{aligned}
$$

which gives

$$
\int_{|x|=r} e_{\alpha}\left\langle A \nabla e_{\alpha}, \mathbf{n}\right\rangle d S-\int_{|x|=1}\left\langle A \nabla e_{\alpha}, \mathbf{n}\right\rangle d S=\int_{1<|x|<r}\left\{\left\langle A \nabla e_{\alpha}, \nabla e_{\alpha}\right\rangle+\alpha e_{\alpha}^{2} b\right\} d x
$$


for a.a. $r>1$. Consequently, we see that

$$
V_{\alpha}(r) \stackrel{\text { def }}{=} \int_{|x|>r}\left\{\left\langle A \nabla e_{\alpha}, \nabla e_{\alpha}\right\rangle+\alpha e_{\alpha}^{2} b\right\} d x=-\int_{|x|=r} e_{\alpha}\left\langle A \nabla e_{\alpha}, \mathbf{n}\right\rangle d S
$$

for a.a. $r>1$. We now compute the upper bound for $V_{\alpha}(r)$.

$$
\begin{aligned}
V_{\alpha}(r) & \leq \int_{|x|=r} \sqrt{\left\langle A \nabla e_{\alpha}, \nabla e_{\alpha}\right\rangle} \sqrt{\langle A \mathbf{n}, \mathbf{n}\rangle} e_{\alpha} d S \\
& =\int_{|x|=r} 2 \sqrt{\left\langle A \nabla e_{\alpha}, \nabla e_{\alpha}\right\rangle} \sqrt{\alpha e_{\alpha}^{2} b} d S \frac{1}{2 \sqrt{\alpha}} \sqrt{B_{+}(r)} \\
& \leq \frac{\sqrt{B_{+}(r)}}{2 \sqrt{\alpha}} \int_{|x|=r}\left\{\left\langle A \nabla e_{\alpha}, \nabla e_{\alpha}\right\rangle+\alpha e_{\alpha}^{2} b\right\} d S \\
& =-\frac{d V_{\alpha}(r)}{d r} \frac{\sqrt{B_{+}(r)}}{2 \sqrt{\alpha}} \text { for a.a. } r>1 .
\end{aligned}
$$

Solving this differential inequality, we obtain

$$
V_{\alpha}(r) \leq V_{\alpha}(1) \exp \left\{-\int_{1}^{r} \frac{2 \sqrt{\alpha}}{\sqrt{B_{+}(s)}} d s\right\} \text { for all } r>1 .
$$

On the other hand, as for the lower bound, we have

$$
V_{\alpha}(r) \geq \int_{|x|>r}\left\langle A \nabla e_{\alpha}, \nabla e_{\alpha}\right\rangle d x=\int_{r}^{+\infty} d s \int_{|x|=s}\left\langle A \nabla e_{\alpha}, \nabla e_{\alpha}\right\rangle d S .
$$

The Schwarz inequality gives

$$
\int_{|x|=s}\left\langle A \nabla e_{\alpha}, \nabla e_{\alpha}\right\rangle d S \int_{|x|=s}\langle A \mathbf{n}, \mathbf{n}\rangle d S \geq\left[\int_{|x|=s}\left\langle A \nabla e_{\alpha}, \mathbf{n}\right\rangle d S\right]^{2} .
$$

Combining all of this, we obtain

$$
V_{\alpha}(1) \exp \left\{-\int_{1}^{r} \frac{2 \sqrt{\alpha}}{\sqrt{B_{+}(s)}} d s\right\} \geq \int_{r}^{+\infty}\left[\int_{|x|=s}\left\langle A \nabla e_{\alpha}, \mathbf{n}\right\rangle d S\right]^{2} s^{1-n} A_{+}(s)^{-1} d s .
$$

By the previous argument on $a_{\alpha}$, we see that

$$
\left|a_{\alpha}\right| \leq\left|\int_{|x|=s}\left\langle A \nabla e_{\alpha}, \mathbf{n}\right\rangle d S\right| \text { for a.a. } s>1 .
$$

Thus we finally obtain the inequality

$$
\left|a_{\alpha}\right|^{2} \leq \frac{V_{\alpha}(1) \exp \left\{-\int_{1}^{r} 2 \sqrt{\alpha} / \sqrt{B_{+}(s)} d s\right\}}{\int_{r}^{+\infty} s^{1-n} A_{+}(s)^{-1} d s},
$$

which tends to zero as $r \rightarrow+\infty$ by the assumption. This completes the proof of Theorem 1. Q.E.D.

We now turn to sufficient conditions on the coefficients $a_{i j}$ and $b$ for the explosion of the diffusion. We have the following theorem. 
THEOREM 3.2. If

$$
\int_{S^{n-1}} d \sigma \int_{1}^{+\infty} r^{1-n}\left\langle A(r \sigma)^{-1} \sigma, \sigma\right\rangle\left[\int_{1}^{r} s^{n-1} b(s \sigma) d s\right]^{2} d r<+\infty,
$$

then the explosion is sure. Here $A(x)^{-1}$ is the inverse matrix of $A(x)$.

We first note that according to Ichihara $[6], X_{t}$ is transient under the assumption in Theorem 3.2. In order to prove Theorem 3.2, we shall verify the condition in Theorem B.

PROOF OF THEOREM 3.2. Introduce the following hitting time:

$$
\sigma_{\rho}=\left\{\begin{array}{ll}
\inf \left\{t \geq 0|| X_{t} \mid \geq \rho\right\} & \text { if such } t \text { exists } \\
+\infty & \text { otherwise }
\end{array} \text { for } \rho>1 .\right.
$$

Define $e_{0}^{\rho}(x)=P_{x}\left[\tau_{1}<\sigma_{\rho}\right]$. It is obvious that $e_{0}^{\rho}(x)$ is monotone nondecreasing and is convergent to $e_{0}(x)$ as $\rho \uparrow+\infty$. Thus we have

$$
\lim _{\rho \uparrow+\infty} \int_{\rho>|x|>1} e_{0}^{\rho}(x) b(x) d x=\int_{|x|>1} e_{0}(x) b(x) d x
$$

by virtue of the monotone convergence theorem. We now estimate, by polar coordinates,

$$
\begin{aligned}
\int_{\rho>|x|>1} e_{0}^{\rho}(x) b(x) d x & =\int_{S^{n-1}} d \sigma \int_{1}^{\rho} r^{n-1} b(r \sigma) e_{0}^{\rho}(r \sigma) d r \\
& =\int_{S^{n-1}} d \sigma \int_{1}^{\rho} r^{n-1} b(r \sigma) d r \int_{r}^{\rho}-\frac{\partial e_{0}^{\rho}}{\partial s}(s \sigma) d s \\
& \leq \int_{S^{n-1}} d \sigma \int_{1}^{\rho}\left|\frac{\partial e_{0}^{\rho}(s \sigma)}{\partial s}\right| d s \int_{1}^{s} r^{n-1} b(r \sigma) d r \\
& =\int_{S^{n-1}} d \sigma \int_{1}^{\rho}\left|\left\langle\sqrt{A} \nabla e_{0}^{\rho}, \sqrt{A}^{-1} \mathbf{n}\right\rangle(s \sigma)\right| d s \int_{1}^{s} r^{n-1} b(r \sigma) d r
\end{aligned}
$$

(where $\sqrt{A}$ is a symmetric positive square $\operatorname{root}$ of $A$ )

$$
\begin{aligned}
\leq & \int_{S^{n-1}} d \sigma \sqrt{\int_{1}^{\rho}\left\langle A \nabla e_{0}^{\rho}, \nabla e_{0}^{\rho}\right\rangle(s \sigma) s^{n-1} d s} \\
& \times \sqrt{\int_{1}^{\rho}\left\langle A^{-1} \mathbf{n}, \mathbf{n}\right\rangle(s \sigma) s^{1-n}\left[\int_{1}^{s} r^{n-1} b(r \sigma) d r\right]^{2}} d s \\
\leq & \sqrt{\int_{1<|x|<\rho}\left\langle A \nabla e_{0}^{\rho}, \nabla e_{0}^{\rho}\right\rangle d x} \\
& \times \sqrt{\int_{S^{n-1}} d \sigma \int_{1}^{\rho}\left\langle A^{-1} \mathbf{n}, \mathbf{n}\right\rangle(s \sigma) s^{1-n}\left[\int_{1}^{s} r^{n-1} b(r \sigma) d r\right]^{2}} d s \\
\leq & \sqrt{\mathcal{E}_{0}\left(e_{0}^{\rho}, e_{0}^{\rho}\right)} \sqrt{\int_{S^{n-1}} d \sigma \int_{1}^{\rho}\left\langle A^{-1} \mathbf{n}, \mathbf{n}\right\rangle(s \sigma) s^{1-n}\left[\int_{1}^{s} r^{n-1} b(r \sigma) d r\right]^{2}} d s .
\end{aligned}
$$

A simple application of the Dirichlet principle gives

$$
\lim _{\rho \rightarrow+\infty} \mathcal{E}_{0}\left(e_{0}^{\rho}, e_{0}^{\rho}\right)=\mathcal{E}_{0}\left(e_{0}, e_{0}\right)=C_{0}\left(B_{n}\right)
$$


(see Ichihara [6]). Thus we obtain

$$
\begin{aligned}
\int_{|x|>1} e_{0}(x) b(x) d x \leq & \sqrt{C_{0}\left(B_{n}\right)} \\
& \times \sqrt{\int_{S^{n-1}} d \sigma \int_{1}^{+\infty}\left\langle A(s \sigma)^{-1} \sigma, \sigma\right\rangle s^{1-n}\left[\int_{1}^{s} r^{n-1} b(r \sigma) d r\right]^{2}} d s
\end{aligned}
$$

which is finite by the hypothesis in Theorem 3.2. This completes the proof. Q.E.D.

Hunt [5] has given an upper bound for the hitting probability of the unit sphere centered at the origin by $X_{t}$. Define

$$
A_{-}(r)=\inf _{\substack{|x|=r \\|\xi|=1}}\langle A(x) \xi, \xi\rangle .
$$

Then he has shown that with a positive constant $C$,

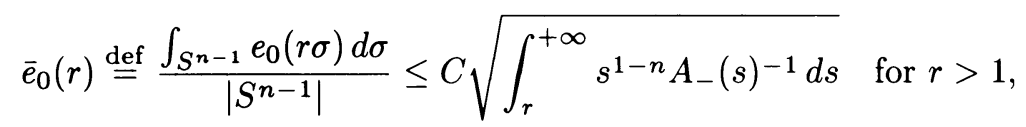

where $\left|S^{n-1}\right|$ is the surface area of $S^{n-1}$. Define

$$
b_{-}(r)=\sup _{|x|=r} b(x) .
$$

Theorem $\mathrm{B}$ together with the above upper bound for $e_{0}$ gives the following theorem.

THEOREM 3.3. If

$$
\int_{1}^{+\infty} r^{n-1} b_{-}(r) \sqrt{\int_{r}^{+\infty} s^{1-n} A_{-}(s)^{-1} d s} d r<+\infty
$$

then the explosion of $X_{t}$ is sure.

In order to illustrate Theorems 3.1 and 3.2 , we shall exhibit some examples.

EXAMPLE 3.1. Consider the case $b=1$ on $\mathbf{R}^{n}$. If $a_{i j}(x)=O\left(|x|^{2}\right)$, then the process $X_{t}$ is conservative. Indeed, it holds under the conditions that with positive constants $C_{1}$ and $C_{2}, A_{+}(r) \leq C_{1} r^{2}$ and $B_{+}(r) \leq C_{2} r^{2}$. Consequently, we have

$$
\begin{aligned}
\frac{\exp \left\{-\int_{1}^{r} 2 \sqrt{\alpha} / \sqrt{B_{+}(s)} d s\right\}}{\int_{r}^{+\infty} s^{1-n} A_{+}(s)^{-1} d s} & \leq \frac{\exp \left\{-\int_{1}^{r} 2 \sqrt{\alpha} / \sqrt{C_{2}} d s / s\right\}}{\int_{r}^{+\infty} s^{1-n} d s / C_{1} s^{2}} \\
& =\text { const } \times r^{n-2 \sqrt{\alpha / C_{1}}} \rightarrow 0, \text { as } r \rightarrow+\infty
\end{aligned}
$$

for any positive $\alpha$ with $n<2 \sqrt{\alpha / C_{1}}$. This shows that $X_{t}$ is conservative.

EXAMPLE 3.2. Let us assume that $X_{t}$ is associated with the operator

$$
L=\frac{1}{a(x)} \sum_{i=1}^{n} \frac{\partial}{\partial x_{i}}\left(a(x) \frac{\partial}{\partial x_{i}}\right) .
$$

Let $\bar{a}(r)=\int_{S^{n-1}} a(r \sigma) d \sigma$. If $\bar{a}(r)$ satisfies

$$
\varliminf_{r \rightarrow+\infty} \frac{\log \left[\int_{r}^{+\infty} \frac{d s}{s^{n-1} \bar{a}(s)}\right]}{r}>-\infty
$$


then the diffusion $X_{t}$ is conservative. In particular, if $\bar{a}(r) \leq C_{1} e^{C_{2} r}$ holds with positive constants $C_{1}$ and $C_{2}$, then the above condition is satisfied. Since $A_{+}(r)=$ $\bar{a}(r)$ and $B_{+}(r)=1$, it is easy to verify the condition in Theorem 3.1.

In the following example, it is shown that the second case in Theorem $\mathrm{C}$ can certainly occur.

EXAMPLE 3.3. Let

$$
L=\frac{\partial}{\partial x_{1}}\left(a\left(x_{1}\right) \frac{\partial}{\partial x_{1}}\right)+\frac{\partial^{2}}{\partial x_{2}^{2}} .
$$

Suppose

$$
\int_{0}^{+\infty} a\left(x_{1}\right)^{-1} d x_{1}<+\infty, \quad \int_{0}^{+\infty} x_{1} a\left(x_{1}\right)^{-1} d x_{1}=+\infty
$$

and

$$
\int_{-\infty}^{0} x_{1} a\left(x_{1}\right)^{-1} d x_{1}>-\infty
$$

Then we have

$$
\begin{aligned}
& 0<P_{\left(x_{1}, x_{2}\right)}[\zeta=+\infty]<1, \\
& \lim _{x_{1} \rightarrow-\infty} P_{\left(x_{1}, x_{2}\right)}[\zeta=+\infty]=0,
\end{aligned}
$$

and

$$
\lim _{x_{1} \rightarrow+\infty} P_{\left(x_{1}, x_{2}\right)}[\zeta=+\infty]=1 .
$$

Indeed, the first component $X_{t}^{1}$ of $X_{t}$ is regarded as the one-dimensional diffusion process corresponding to the operator

$$
\frac{d}{d x_{1}}\left(a\left(x_{1}\right) \frac{d}{d x_{1}}\right) \text {. }
$$

$-\infty$ is an exit boundary for the diffusion $X_{t}^{1}$. Though $+\infty$ is a natural boundary for $X_{t}^{1}$, the probability that $X_{t}^{1}$ approaches $+\infty$ is still positive from the assumption. The second component $X_{t}^{2}$ is obviously a standard Brownian motion on $\mathbf{R}^{\mathbf{1}}$. Combining these, we obtain the above assertions.

EXAMPLE 3.4. Let

$$
L=\sum_{i=1}^{n} \frac{\partial}{\partial x_{i}}\left(a_{i}(x) \frac{\partial}{\partial x_{i}}\right) .
$$

Suppose that with some positive constants $C$ and $\alpha>1$,

$$
a_{i}(x) \geq C|x|^{n+2}(\log |x|)^{\alpha}
$$

for all sufficiently large $x \in \mathbf{R}^{n}$. Then it is easy to verify that the condition for the explosion is satisfied.

EXAMPLE 3.5. Let $n \geq 3$ and $a_{i j}(x)=\delta_{i j}, i, j=1, \ldots, n$, where

$$
\delta_{i j}= \begin{cases}1, & \text { if } i=j, \\ 0, & \text { otherwise. }\end{cases}
$$

If the condition

$$
\int_{|x|>1} \frac{b(x) d x}{|x|^{n-2}}<+\infty
$$


is satisfied, then the explosion is sure. Indeed, the hitting probability $e_{0}$ is given by $e_{0}(x)=1 /|x|^{n-2}$ for $|x| \geq 1$. Consequently, from Theorem B, we obtain the above results.

Up to now, we have worked with diffusion processes associated with strictly elliptic differential operators with measurable coefficients. If the smoothness of the coefficients is assumed, then the strict ellipticity is considerably relaxed. Indeed, suppose the coefficients $a_{i j}$ and $b$ are infinitely differentiable. Define first-order differential operators $X_{i}$ by

$$
X_{i}=\sum_{j=1}^{n} a_{i j} \frac{\partial}{\partial x_{j}}, \quad i=1, \ldots, n .
$$

It is well known that the operator $L$ is hypoelliptic if the Lie algebra generated by $X_{1}, X_{2}, \ldots, X_{n}$ has the full rank in $\mathbf{R}^{n}$, i.e. $\operatorname{rank} \mathcal{L}\left(X_{1}, \ldots, X_{n}\right)_{x}=n, x \in \mathbf{R}^{n}$. See Oleinik and Radkevich [14]. In this case we can prove the same results for the process as in $\S \S 2$ and 3.

4. Singular cases. In this section we shall investigate conservativeness and explosion of diffusion processes associated with Dirichlet forms endowed with measures $\nu_{i j}, i, j=1, \ldots, n$, and $m$ not necessarily absolutely continuous with respect to the Lebesgue measure $d x$. We shall study the explosion properties for the following two particular cases.

[I] Time-changed processes. Suppose that $\nu_{i j}(d x), i, j=1, \ldots, n$, are absolutely continuous with respect to $d x$, i.e. $\nu_{i j}(d x)=a_{i j}(x) d x$. However the speed measure $m(d x)$ may possibly be singular. Let $m(d x)=m_{s}(d x)+b(x) d x$ be the Lebesgue decomposition where $m_{s}$ is singular with respect to $d x$. Let us assume the conditions (C.1) and (C.2) for the coefficients $a_{i j}$ and $b$. We have the following.

REMARK 4.1. It is easy to see under the above conditions (C.1) and (C.2) that the symmetric form on $L^{2}\left(\mathbf{R}^{n}, m\right)$ defined by

$$
\mathcal{E}(u, v)=\int_{\mathbf{R}^{n}} \sum_{i, j=1}^{n} a_{i j} \frac{\partial u}{\partial x_{i}} \frac{\partial v}{\partial x_{j}} d x \quad \text { for } u, v \in C_{0}^{\infty}\left(\mathbf{R}^{n}\right)
$$

is closable. Indeed, suppose that $u_{k, k \geq 1} \in C_{0}^{\infty}\left(\mathbf{R}^{n}\right)$ satisfy

$$
\lim _{k, l \rightarrow+\infty} \mathcal{E}\left(u_{k}-u_{l}, u_{k}-u_{l}\right)=0
$$

and

$$
\lim _{k \rightarrow 0}\left(u_{k}, u_{k}\right)=0 .
$$

Then we see that there exists a unique element $\left(g_{i}\right)_{i=1, \ldots, n}, g_{i} \in L_{\text {loc }}^{2}\left(\mathbf{R}^{n}, d x\right)$, to which $\nabla u_{k}=\left(\partial u_{k} / \partial x_{i}\right)_{i=1, \ldots, n}$ converges in $L_{\text {loc }}^{2}\left(\mathbf{R}^{n}, d x\right)$, and that $u_{k}$ also converges to zero in $L_{\text {loc }}^{2}\left(\mathbf{R}^{n}, d x\right)$. For any $\phi$ in $C_{0}^{\infty}\left(\mathbf{R}^{n}\right)$,

$$
\int_{\mathbf{R}^{n}} \frac{\partial u_{k}}{\partial x_{i}} \phi d x=-\int_{\mathbf{R}^{n}} u_{k} \frac{\partial \phi}{\partial x_{i}} d x
$$

Letting $k$ tend to infinity, we obtain

$$
\int_{\mathbf{R}^{n}} g_{i} \phi d x=0 \quad \text { for all } \phi \in C_{0}^{\infty}\left(\mathbf{R}^{n}\right),
$$


which shows $g_{i}=0$ a.e. on $\mathbf{R}^{n}, i=1, \ldots, n$. Subtracting a subsequence if necessary, we may assume that $\partial u_{k} / \partial x_{i}, i=1, \ldots, n$, converges to zero a.e. on $\mathbf{R}^{n}$. Then, by Fatou's lemma, we have

$$
\begin{aligned}
\mathcal{E}\left(u_{k}, u_{k}\right) & =\int_{\mathbf{R}^{n}} \lim _{l \rightarrow+\infty} \sum_{i, j=1}^{n} a_{i j} \frac{\partial\left(u_{l}-u_{k}\right)}{\partial x_{i}} \frac{\partial\left(u_{l}-u_{k}\right)}{\partial x_{j}} d x \\
& \leq \underset{l \rightarrow+\infty}{\lim } \mathcal{E}\left(u_{k}-u_{l}, u_{k}-u_{l}\right),
\end{aligned}
$$

which can be made arbitrarily small for a sufficiently large $k$.

From Remark 4.1, we see that there exists a diffusion process $\left(X_{t}, \zeta, P_{x}\right)$ whose Dirichlet form is the closed extension $\overline{\mathcal{E}}$ of $\mathcal{E}$. Furthermore let us introduce the unique minimal diffusion process $\left(X_{t}^{0}, \varsigma^{0}, P_{x}^{0}\right), x \in \mathbf{R}^{n}$, corresponding to the operator

$$
L^{0}=\sum_{i, j=1}^{n} \frac{\partial}{\partial x_{i}}\left(a_{i j} \frac{\partial}{\partial x_{j}}\right) .
$$

We assume the following condition for the diffusion $X_{t}^{0}$.

(C.3) $X_{t}^{0}$ is transient.

See Ichihara [6] for the transience of the diffusion $X_{t}^{0}$. Let $C_{0}^{0}$ and $C_{0}$ denote the 0-order capacities with respect to $X_{t}^{0}$ and $X_{t}$ respectively. We need an additional condition.

(C.4) The speed measure $m$ charges no set of $C_{0}^{0}$-capacity zero.

Under the conditions (C.1)-(C.4), Theorem 5.5.2 in Fukushima [2] asserts that the diffusion $X_{t}$ is equivalent to the time-changed process with the speed measure $m$ of the process $X_{t}^{0}$. The capacities $C_{0}^{0}$ and $C_{0}$ are the same. Let $G_{0}^{0}(x, y)$ be the 0 -order Green function of $X_{t}^{0}$ and $G_{0}(x, d y)$ be the 0-order Green measure of $X_{t}$. Then we have the following relation between them.

$$
G_{0}(x, d y)=G_{0}^{0}(x, y) m(d y)
$$

for q.e. $x$ in $\mathbf{R}^{n}$. We are now in a position to state our theorems for the above diffusion $X_{t}$.

THEOREM $\mathrm{A}^{\prime}$. The following conditions are equivalent.

(1) $X_{t}$ is conservative, i.e. $P_{x}[\varsigma=+\infty]=1$ for q.e. $x$ in $\mathbf{R}^{n}$.

(2) For some $\alpha>0$,

$$
\int_{\mathbf{R}^{n}} \alpha e_{\alpha}(x) m(d x)=C_{\alpha}\left(B_{n}\right)
$$

(3) For all $\alpha>0$,

$$
\int_{\mathbf{R}^{n}} \alpha e_{\alpha}(x) m(d x)=C_{\alpha}\left(B_{n}\right) .
$$

THEOREM $\mathrm{B}^{\prime}$. If $\int_{\mathbf{R}^{n}} e_{0}(x) m(d x)<+\infty$, then $X_{t}$ starting at q.e. $x$ in $\mathbf{R}^{n}$ explodes with probability one, i.e.,

$$
P_{x}[\varsigma<+\infty]=1 \text { for q.e. } x \in \mathbf{R}^{n} .
$$

ProOF OF THEOREM $\mathrm{A}^{\prime}$. In the proof of Theorem $\mathrm{A}$, we have shown

$$
\int_{\mathbf{R}^{n}} \alpha e_{\alpha}(x) m(d x)=\int_{B^{n}} \alpha G_{\alpha} 1(x) \mu_{\alpha}(d x) .
$$


Suppose condition (1) holds. Then $\alpha G_{\alpha} 1(x)=1$ for q.e. $x \in \mathbf{R}^{n}$. Theorem 3.3.2, Fukushima [2], implies $\mu_{\alpha}\left(\left\{x \in B_{n} \mid \alpha G_{\alpha} 1(x)<1\right\}\right)=0$, which gives

$$
\int_{\mathbf{R}^{n}} \alpha e_{\alpha}(x) m(d x)=\int_{B^{n}} \alpha G_{\alpha} 1(x) \mu_{\alpha}(d x)=\mu_{\alpha}\left(B_{n}\right)=C_{\alpha}\left(B_{n}\right) .
$$

Thus conditions (2) and (3) follow from (1). Conversely, suppose (2) is satisfied. We first note that (4.1) implies

(H.1)' For each $\alpha>0, G_{\alpha}(x, \cdot)$, q.e. $x \in \mathbf{R}^{n}$ are equivalent to each other. It follows from (2) that $P_{x}[\zeta=+\infty]=1$ for $\mu_{\alpha}$-a.a. $x$ on $B_{n}$. Since

$$
\mu_{\alpha}\left(\left\{x \in B_{n} \mid P_{x}[\zeta=+\infty]=1\right\}\right)=C_{\alpha}\left(B_{n}\right)>0,
$$

by Theorem 3.3.2, Fukushima [2], we can choose a point $x_{0} \in B_{n}$ such that

$$
P_{x_{0}}[\zeta=+\infty]=1
$$

and

$$
\text { for q.e. } x \in \mathbf{R}^{n}, G_{\alpha}(x, \cdot) \text { is equivalent to } G_{\alpha}\left(x_{0}, \cdot\right) \text {. }
$$

With the help of the above conditions (4.2) and (4.3), we can prove the implication $(2) \Rightarrow(1)$. Q.E.D.

PROOF OF THEOREM $\mathrm{B}^{\prime}$. As was shown in $\S 3$,

$$
\int_{\mathbf{R}^{n}} e_{0}(x) m(d x)=\int_{\partial B_{n}} E_{x}[\zeta] \mu_{0}(d x) .
$$

Thus $P_{x}[\varsigma<+\infty]=1$ for $\mu_{0}$-a.e. $x$ on $\partial B_{n}$. Since $\mu_{0}\left(\left\{x \in \partial B_{n} \mid P_{x}[\varsigma<+\infty]=\right.\right.$ 1\}) $=C_{0}\left(B_{n}\right)>0$, by Theorem 3.3.2, Fukushima [2], we can choose a point $x_{0} \in \partial B_{n}$ satisfying

$$
P_{x_{0}}[\zeta<+\infty]=1
$$

and (4.3). With the help of (4.3) and (4.4), we can prove the assertion by the same argument as in the proof of Theorem B. Q.E.D.

Let $A(x)=\left(a_{i j}(x)\right)_{i, j=1, \ldots, n}$ and $A_{+}(r)$ denote the same as in the preceeding section. Define $B_{+}(r)$ in terms of $A(x)$ and $b(x)$, the Radon-Nikodým derivative of the absolutely continuous part of $m(d x)$, as in $\S 3$. By means of Theorems $\mathrm{A}^{\prime}$ and $\mathrm{B}^{\prime}$, we can prove the following conditions on $a_{i j}$ and $b$ for conservativeness and explosion.

THEOREM 4.1. If for some $\alpha>0$,

$$
\lim _{r \rightarrow+\infty} \frac{\exp \left\{-\int_{1}^{r} 2 \sqrt{\alpha} / \sqrt{B_{+}(s)} d s\right\}}{\int_{r}^{+\infty} s^{1-n} A_{+}(s)^{-1} d s}=0
$$

then the diffusion $X_{t}$ is conservative, i.e.

$$
P_{x}[\zeta=+\infty]=1 \text { for q.e. } x \in \mathbf{R}^{n} .
$$

THEOREM 4.2. Let $n \geq 3$ and the operator $L^{0}$ be uniformly elliptic, i.e. with some positive constant $\lambda>1$,

$$
\lambda^{-1} E_{n} \leq\left(a_{i j}(x)\right)_{i, j=1, \ldots, n} \leq \lambda E_{n},
$$


where $E_{n}$ is the identity matrix. If

$$
\int_{|x|>1} \frac{m(d x)}{|x|^{n-2}}<+\infty
$$

then the diffusion starting at q.e. $x \in \mathbf{R}^{n}$ explodes with probability one.

See McKean and Tanaka [12] for relevant results.

THEOREM 4.3. Suppose that the speed measure $m(d x)$ is rotationally symmetric about the origin, i.e. $m(d x)=m_{0}(d r) d \sigma, x=(r, \sigma) \in[0,+\infty) \times S^{n-1}$. If

$$
\int_{1}^{+\infty} \sqrt{\int_{r}^{+\infty} s^{1-n} A_{-}(s)^{-1} d s m_{0}}(d r)<+\infty,
$$

then the diffusion starting at q.e. $x \in R^{n}$ explodes with probability one.

The proof of Theorem 4.1 is carried out in exactly the same way as in Theorem 3.1. For the proof of Theorem 4.2, we need the following

LEMMA 4.1. (See Littman et al. [10].) Let $n \geq 3$. Suppose $L^{0}$ is uniformly elliptic. Then the 0-order Green operator $G_{0}^{0}(x, y)$ satisfies the following inequality: for some positive constant $\lambda>1$,

$$
G_{0}^{0}(x, y) \leq \frac{\lambda}{|x-y|^{n-2}}, \quad x, y \in \mathbf{R}^{n} .
$$

Some potential-theoretic considerations together with Lemma 4.1 give the following upper bound for the hitting probability of the unit sphere $e_{0}(x)$ : with a positive constant $\lambda_{0}$,

$$
e_{0}(x) \leq \frac{\lambda_{0}}{|x|^{n-2}} \quad \text { for } x \in \mathbf{R}^{n}
$$

See, for example, Ichihara and Watanabe [8]. Theorem $B^{\prime}$ together with the above upper bound for $e_{0}(x)$ verifies Theorem 4.2. Theorem 4.3 follows from Theorem $\mathrm{B}^{\prime}$ and Hunt's estimate for the hitting probability of the unit sphere by the diffusion.

We shall exhibit some examples of speed measures which charge no set of $C_{0^{-}}^{0}$ capacity zero. The following lemma is useful in the construction of examples.

LEMMA 4.2. If a positive Radon measure $m$ on $\mathbf{R}^{n}, n \geq 2$, satisfies

$$
\begin{aligned}
\iint_{\mathbf{R}^{n} \times \mathbf{R}^{n}} \frac{m(d x) m(d y)}{|x-y|^{n-2}}<+\infty & \text { for } n \geq 3, \\
\iint_{\mathbf{R}^{2} \times \mathbf{R}^{2}}|\log | x-y|| m(d x) m(d y)<+\infty & \text { for } n=2,
\end{aligned}
$$

then $m$ charges no set of $C_{0}^{0}$-capacity zero.

This lemma follows from Kelvin's principle (see, for example, Itô and McKean [9]), because the capacity $C_{0}^{0}$ is equivalent to the corresponding capacity with respect to a standard Brownian motion.

EXAMPLE 4.1. Let $m(d x)=d x_{1} d x_{2} \cdot \ldots \cdot d x_{n-1} m_{0}\left(d x_{n}\right)$ where $m_{0}(d t)$ is a positive Radon measure on $\mathbf{R}^{1}$. Then the measure $m$ on $\mathbf{R}^{n}$ charges no set of 
$C_{0}^{0}$-capacity zero. Indeed, it is easy to see that for each compact set $K \subset \mathbf{R}^{n}$, the function defined by

$$
\begin{cases}\int_{K} \frac{m(d y)}{|x-y|^{n-2}} & \text { for } n \geq 3 \\ \int_{K}\left[\log \frac{1}{|x-y|}\right] m(d y) & \text { for } n=2\end{cases}
$$

is continuous in $x$. This implies that the measure $m$ restricted to $K$ satisfies the assumption in Lemma 4.2 .

EXAMPLE 4.2. Let $a_{k}, k \geq 1$, be a sequence of increasing positive numbers. Define $m(d x)$ on $\mathbf{R}^{n}$ by

$$
m(d x)=\sum_{k=1}^{\infty} \delta_{a_{k}}(d r) d \sigma, \quad x=(r, \sigma) \in(0,+\infty) \times S^{n-1} .
$$

Then $m$ charges no set of $C_{0}^{0}$-capacity zero.

[II] Singular energy measures. Here we assume $m(d x)=d x$, Lebesgue measure. Let $\nu(d t)$ be a positive Radon measure on $\mathbf{R}^{1}$ strictly positive for each open interval. Let $\nu(d t)=\nu_{s}(d t)+a_{0}(t) d t$ be the Lebesgue decomposition where $\nu_{s}$ is singular with respect to $d t$. Suppose

$$
\inf \left\{a_{0}(t), t \in U\right\}>0
$$

for every nonempty bounded open set $U \subset \mathbf{R}^{1}$. Define positive Radon measures $\tilde{\nu}_{i j}(d x)=\tilde{\nu}_{i j}\left(d x_{1} \ldots d x_{n}\right), i, j=1, \ldots, n$, on $\mathbf{R}^{n}$ by

$$
\tilde{\nu}_{i j}(d x)= \begin{cases}d x_{1} \ldots d x_{n-1} \nu\left(d x_{n}\right) & \text { if } i, j=1, \ldots, n-1, \\ d x_{1} \ldots d x_{n-1} d x_{n} & \text { otherwise. }\end{cases}
$$

Let $a_{i j}, i, j=1, \ldots, n$, be measurable functions such that $a_{i j}=a_{j i}$ and such that for any compact set $K \subset \mathbf{R}^{n}$, there is a positive constant $\lambda=\lambda(K)>1$ such that

$$
\lambda^{-1} \sum_{i=1}^{n} \xi_{i}^{2} \tilde{\nu}_{i i}(d x) \geq \sum_{i, j=1}^{n} \xi_{i} \xi_{j} a_{i j}(x) \tilde{\nu}_{i j}(d x) \leq \lambda \sum_{i=1}^{n} \xi_{i}^{2} \tilde{\nu}_{i i}(d x)
$$

for all $(x, \xi) \in K \times \mathbf{R}^{n}$. Set $\nu_{i j}(d x)=a_{i j}(x) \tilde{\nu}_{i j}(d x)$ and consider the bilinear symmetric form

$$
\mathcal{E}(u, v)=\int_{\mathbf{R}^{n}} \frac{\partial u}{\partial x_{i}} \frac{\partial v}{\partial x_{j}} \nu_{i j}(d x)
$$

on $L^{2}\left(\mathbf{R}^{n}, d x\right)$. From now on, we assume

(C.5) $a_{i j}$ is of the $C^{n_{0}}$-class on $\mathbf{R}^{n}$, where $n_{0}=[(n-1) / 2]+1$.

Tomisaki [15] has shown under the above conditions that three exists a unique diffusion process $\left(X_{t}, \zeta, P_{x}\right), x \in \mathbf{R}^{n}$, whose Dirichlet form is the closed extension $\bar{\varepsilon}$ of $\mathcal{E}$, and whose resolvent $R_{\lambda}$ is continuous in the sense that for each $f \in L^{2}\left(\mathbf{R}^{n}, d x\right) \cap L^{p}\left(\mathbf{R}^{n}, d x\right)$ with $p>n, \mathbf{R}_{\lambda} f(x)$ is continuous in $\mathbf{R}^{n}$. It is obvious from Theorem 9.2, Tomisaki [16], that the hypothesis (H.1) is satisfied for this process. Thus, in this case, Theorems A and B are valid. Define

$$
\begin{aligned}
\tilde{a}_{i j}(x) & = \begin{cases}a_{i j}\left(x_{1}, \ldots, x_{n}\right) a_{0}\left(x_{n}\right) & \text { if } i, j=1, \ldots, n-1, \\
a_{i j}\left(x_{1}, \ldots, x_{n}\right) & \text { otherwise },\end{cases} \\
\tilde{A}(x) & =\left(\tilde{a}_{i j}(x)\right)_{i, j=1, \ldots, n},
\end{aligned}
$$


and

$$
\tilde{A}_{-}(r)=\inf _{\substack{|\xi|=1 \\|x|=r}}\langle\tilde{A}(x) \xi, \xi\rangle, \quad r>0 .
$$

The following theorem gives a sufficient condition on the coefficients $a_{i j}$ and $a_{0}$ for the explosion of the diffusion.

THEOREM 4.4. If

$$
\int_{1}^{+\infty} r^{n-1} \sqrt{\int_{r}^{+\infty} s^{1-n} \tilde{A}_{-}(s)^{-1} d s} d r<+\infty
$$

then the diffusion $X_{t}$ explodes with probability one, i.e.

$$
P .[\varsigma<+\infty]=1 \text { on } \mathbf{R}^{n} \text {. }
$$

Theorem 4.4 follows from Theorem B combined with the following lemma.

LEMMA 4.3. Let $e_{0}(x)$ be the hitting probability of the unit sphere by the diffusion $X_{t}$. Then, for $r>1$,

$$
\bar{e}_{0}(r) \stackrel{\text { def }}{=} \frac{\int_{S^{n-1}} e_{0}(r \sigma) d \sigma}{\left|S^{n-1}\right|} \leq \mathrm{const} \sqrt{\int_{r}^{+\infty} s^{1-n} \tilde{A}_{-}(s)^{-1} d s .}
$$

PROOF. The proof follows the same line as in Hunt [5], which is presented here. Let $e_{0}^{\rho}$ be the same as in the proof of Theorem 3.2. Note that

$$
\lim _{\rho \rightarrow+\infty} e_{0}^{\rho}(x)=e_{0}(x), \quad x \in \mathbf{R}^{n},
$$

and

$$
\lim _{\rho \rightarrow+\infty} \mathcal{E}_{0}\left(e_{0}^{\rho}, e_{0}^{\rho}\right)=\mathcal{E}_{0}\left(e_{0}, e_{0}\right)
$$

We now estimate

$$
\begin{aligned}
\mathcal{E}_{0}\left(e_{0}^{\rho}, e_{0}^{\rho}\right) & =\int_{1<|x|<\rho} \frac{\partial e_{0}^{\rho}}{\partial x_{i}} \frac{\partial e_{0}^{\rho}}{\partial x_{j}} \nu_{i j}(d x) \\
& \geq \int_{1<|x|<\rho} \frac{\partial e_{0}^{\rho}}{\partial x_{i}} \frac{\partial e_{0}^{\rho}}{\partial x_{j}} \tilde{a}_{i j} d x \\
& \geq \int_{r<|x|<\rho} \sum_{i=1}^{n}\left(\frac{\partial e_{0}^{\rho}}{\partial x_{i}}\right)^{2} \tilde{A}_{-}(|x|) d x, \quad r \in(1, \rho), \\
& \geq \int_{r<|x|<\rho} \sum_{i=1}^{n}\left(\frac{\partial \bar{e}_{0}^{\rho}}{\partial x_{i}}\right)^{2} \tilde{A}_{-}(|x|) d x,
\end{aligned}
$$

by an inequality in Hunt [5], where $\bar{e}_{0}^{\rho}(r)=\int S^{n-1} e_{0}^{\rho}(r \sigma) d \sigma /\left|S^{n-1}\right|$,

$$
\begin{aligned}
& =\int_{r}^{\rho}\left|S^{n-1}\right| \tilde{A}_{-}(s) s^{n-1}\left(\frac{d \bar{e}_{0}^{\rho}}{d s}\right)^{2} d s \\
& \geq \frac{\left(\int_{r}^{\rho} \frac{d \bar{e}_{0}^{\rho}}{d s} d s\right)^{2}\left|S^{n-1}\right|}{\int_{r}^{\rho} s^{1-n} \tilde{A}_{-}(s)^{-1} d s}=\frac{\left|S^{n-1}\right| \cdot \bar{e}_{0}^{\rho}(r)^{2}}{\int_{r}^{\rho} s^{1-n} \tilde{A}_{-}(s)^{-1} d s},
\end{aligned}
$$


by the Schwarz inequality. Thus we have

$$
\bar{e}_{0}^{\rho}(r) \leq \sqrt{\varepsilon_{0}\left(e_{0}^{\rho}, e_{0}^{\rho}\right)} \sqrt{\int_{r}^{\rho} s^{1-n} \tilde{A}_{-}(s)^{-1} d s} / \sqrt{\left|S^{n-1}\right|} .
$$

Taking limits in the above inequality, we obtain

$$
\bar{e}_{0}(r) \leq \sqrt{C_{0}\left(B_{n}\right)} \sqrt{\int_{r}^{+\infty} s^{1-n} \tilde{A}_{-}(s)^{-1} d s} / \sqrt{\left|S^{n-1}\right|}
$$

which completes the proof. Q.E.D.

\section{REFERENCES}

1. W. Feller, The parabolic differential equations and the associated semigroups of transformations, Ann. of Math. (2) 55 (1952), 468-519.

2. M. Fukushima, Dirichlet forms and Markov processes, Kodansha, Tokyo, 1980.

3. D. Gilbarg and N. S. Trudinger, Elliptic partial differential equations of second order, 2nd ed., Springer-Verlag, Berlin and New York, 1983.

4. R. Z. Hasminskii, Ergodic properties of recurrent diffusion processes and stabilization of the problem to the Cauchy problem for parabolic equations, Teor. Veroyatnost. i Primenen. 5 (1960), 196-214.

5. G. A. Hunt, On positive Green functions, Proc. Nat. Acad. Sci. U.S.A. 40 (1954), 816-818.

6. K. Ichihara, Some global properties of symmetric diffusion processes, Publ. Res. Inst. Math. Sci. 14 (1978), 441-486.

7. _ Explosion problems for symmetric diffusion processes, Proc. Japan Acad. Ser. A 60 (1984), 243-245.

8. K. Ichihara and H. Watanabe, Double points for diffusion processes, Japan. J. Math. 6 (1980), 267-281.

9. K. Itô and H. P. McKean, Diffusion processes and their sample paths, Springer-Verlag, Berlin and New York, 1965.

10. W. Littman, G. Stampacchia, and H. F. Weinberger, Regular points for elliptic equations with discontinuous coefficients, Ann. Scuola Norm. Sup. Pisa 17 (1963), 43-77.

11. H. P. McKean, Stochastic integrals, Academic Press, New York, 1969.

12. H. P. McKean and H. Tanaka, Additive functionals of the Brownian path, Memoirs Coll. Sci. Univ. Kyoto Ser. A Math. 33 (1961), 479-506.

13. S. Mizohata, The theory of partial differential equations, Cambridge Univ. Press, London, 1973.

14. O. A. Oleinik and E. D. Radkevich, Second order equations with nonnegative characteristic form, Plenum Press, New York and London, 1973.

15. M. Tomisaki, A construction of diffusion processes with singular product measures, $\mathrm{Z}$. Wahrsch. Verw. Gebiete 53 (1980), 51-70.

16. __ Dirichlet forms associated with direct product diffusion processes, Lecture Notes in Math., vol. 923, Springer-Verlag, Berlin and New York, 1981.

Department of Mathematics, College of General Education, Nagoya UniVERSITY, NAGOYA 464, JAPAN 